\title{
Isolation of fungi as biological control agents against water hyacinth (Eichhornia crassipes)
}

\author{
Ekanayake, E.M.M.S., Manage P.M.*, Liyanage, G.Y. \\ Centre for Water Quality and Algae Research, Department of Zoology, University of Sri \\ Jayewardenepura, Sri Lanka \\ *pathmalal@sjp.ac.lk
}

\begin{abstract}
Aquatic Invasive Species (AIS) cause various damages and losses worldwide. In Sri Lanka, Irrigation Department (ID) has to spend more than 55\% from their annual budget to remove AIS from irrigation systems. Among various types of AIS, water hyacinth; Eichhornia crassipes, is one of the major AIS which spread rapidly in most parts of the world as well as in Sri Lankan water bodies. Therefore the present study was focused to find a microorganism as biological control agent to E. crassipes. E. crassipes plants which showed leaf spot, leaf blight, leaf necrosis, lesions on leaf and petioles were collected from environment to isolate fungi. Isolation of fungi was done by standard spread plate method enriched with Potato Dextrose Agar (PDA). In total, 28 fungal species with different morphologies were isolated and each was exposed to pathogenicity test separately. Out of 28 isolates, six fungal species showed pathogenic effects on water hyacinth and they were tentatively identified as Alternaria sp. (F1), Acremonium sp. (F6, F10), Myrothecium sp. (F4, F28), and Fusarium sp. (F25). F1, F4, F6, F10, F25, F28 damaged 21.45\% $\pm 3.45,5.45 \% \pm 1.78,28.45 \% \pm 2.05$, $25.45 \% \pm 3.45, \quad 14.75 \% \pm 3.19,9.43 \% \pm 1.08$ on leaves and $3.29 \% \pm 1.45,13.39 \% \pm 2.82$, $62.14 \% \pm 4.12,9.34 \% \pm 1.58,6.78 \% \pm 2.45,12.34 \% \pm 3.12$ on petioles respectively while control plants which grow under same conditions without applying fungi were remained healthy. F6 was the most pathogenic isolate and disease conditions were appeared as small necrotic spots and then spread into a leaf blight that entirely covered the whole leaf after a maximum of four weeks from incubation. To confirm whether the F6 fungi has any pathogenic effect on alternative crops, F6 mycelia was introduced to Oryza sativa at laboratory conditions following the same experimental procedure and observed any changes of plants for 4 weeks. Any damage was not observed on $O$. sativa at 4 weeks of incubation. Thus, results of the present study reveals that isolated fungal species can be used as effective bio control agent against water hyacinth.
\end{abstract}

Key words: Water hyacinth, Eichhornia crassipes, Fungi, Biological control 